\title{
THE AXIOM OF CHOICE, FIXED POINT THEOREMS, AND INDUCTIVE ORDERED SETS
}

\author{
MILAN R. TASKOVIĆ
}

(Communicated by Andrew M. Odlyzko)

\begin{abstract}
This paper continues the study of the inductiveness of posets in terms of fixed apexes and points. The author proves some new equivalents of the Axiom of Choice, i.e., Zorn's lemma. These statements are of fixed apex type and fixed point type theorems. The paper includes comments about these theorems and presents new characterizations of inductiveness and quasiinductiveness of posets in terms of fixed apexes and fixed points.
\end{abstract}

\section{INTRODUCTION}

We shall first discuss an assumption that appears to be independent of, and yet consistent with (see [G]), the usual logical assumptions regarding classes and correspondences, but whose absolute validity has been seriously questioned by many authors. This is the so-called Axiom of Choice, which has excited more controversy than any other axiom of set theory since its formulation by Zermelo in 1908. In this sense, many results are known in the set theory (see references).

The fixpoint problem for a given mapping $f \mid P$ is very easy to formulate: the question is whether some $\xi \in P$ satisfies $f(\xi)=\xi$. Many problems are reducible to the existence of fixpoints of certain mappings. The question remains whether some statement (of the axiom of choice type) could be equivalently expressed in the fixpoint language as well. The answer is affirmative.

In this paper we prove some equivalents of the Axiom of Choice. These statements are of fixed point type theorems and fixed apex type theorems. Also, this paper presents new characterizations of inductiveness of posets in terms of fixed apexes and fixed point.

Call a poset (partially ordered set) $P$ inductive (chain complete) when every nonempty chain in $P$ has an upper bound (least upper bound, i.e., supremum) in $P$. Also, call a poset $P$ quasi-inductive (quasi-chain complete) when every nonempty well ordered chain has an upper bound (supremum) in $P$.

In [Ta2] we consider the concept of fixed apexes for the mapping $f$ of a

Received by the editors July 7, 1988 and, in revised form, April 12, 1991. Presented at the Department of Mathematics, Indiana University, Bloomington, Indiana (Mathematics Seminars and Colloquia), October, 1987; and at the University of Missouri-Rolla, Rolla, December, 1987.

1991 Mathematics Subject Classification. Primary 05A15, 04A25, 47H10, 54H25.

Key words and phrases. Inductive partially ordered sets, Axiom of Choice, Zorn's lemma, fixed apexes, fixed points. 
poset $P$ into itself. A map $f$ of a partially ordered set $P$ to itself has a fixed apex $u \in P$ if for $u \in P$ there is $v \in P$ such that $f(u)=v$ and $f(v)=u$. Fixed points are clearly fixed apexes and the set of all fixed points can be a proper subset of the set of fixed apexes. On the other hand, $f$ has a fixed apex if and only if $f^{2}:=f(f)$ has a fixed point. For if $f$ has a fixed apex $u \in P$, then $u=f(v)$ and $v=f(u)$, so $f^{2}$ has a fixed point. If the equation $x=f^{2}(x)$ has a solution $\xi=f^{2}(\xi)$ for some $\xi \in P$, then $f$ has fixed apexes $\xi, f(\xi) \in P$ because $\xi=f^{2}(\xi)$ and $f(\xi)=f(\xi)$.

Let $P$ be a partially ordered set and $f$ a mapping from $P$ into $P$. For any $f: P \rightarrow P$ it is natural to consider the following set:

Sub $f(P):=f(P) \cup\{a \in P \mid a=u b C$ for some chain $C$ in $f(P)\}$,

where $u b C$ is an upper bound of $C$.

\section{MAIN Results}

We are now in a position to formulate our main general statement.

Theorem 1 (Axiom of Choice). Let $P$ be a set partially ordered by an ordering relation $\leq$. Then the following statements are equivalent:

(ZL) (Zorn's lemma) Let $P$ be an inductive poset. Then $P$ has a maximal element.

(FA) [Ta2] Let $P$ be an inductive poset and $f$ a mapping from $P$ into $P$ such that

$$
x \leq f^{2}(x) \quad \text { for all } x \in \operatorname{Sub} f(P) .
$$

Then $f$ has a fixed apex.

(FP) [Ta2] Let $P$ be an inductive poset and $f$ a mapping from $P$ into $P$ such that

$$
x \leq f(x) \quad \text { for all } x \in \operatorname{Sub} f(P) .
$$

Then $f$ has a fixed point.

(ZT) [Z] Let $P$ be a chain complete poset and $f$ a mapping from $P$ into itself such that

(a) there is an element $\theta \in P$ with $\theta \leq x$, for all $x \in P$;

(b) $x \leq f(x)$ for all $x \in P$;

(c) if $x, y \in P$ and $x \leq y \leq f(x)$, then either $x=y$ or $f(x) \leq f(y)$.

Then $f$ has a fixed point.

Proof. We prove the following sequence of implications: $(\mathrm{ZL}) \Rightarrow(\mathrm{FA}) \Rightarrow$ $(\mathrm{FP}) \Rightarrow(\mathrm{ZT}) \Rightarrow(\mathrm{ZL})$.

$(\mathrm{ZL}) \Rightarrow(\mathrm{FA})$. By Zorn's lemma there exists a maximal element $z \in P$, i.e., Sub $f(P)$ has a maximal element $z \in \operatorname{Sub} f(P)$. From condition (M) we have $z \leq f^{2}(z)$ and, because $z$ is a maximal element of set $\operatorname{Sub} f(P), f^{2}(z) \leq z$. Hence, $f^{2}(z)=z$; so from the preceding remarks, $f$ has a fixed apex.

$(\mathrm{FA}) \Rightarrow(\mathrm{FP})$. From (N) we have $f(x) \leq f^{2}(x)$, i.e., $x \leq f(x) \leq f^{2}(x)$ for all $x \in \operatorname{Sub} f(P)$. Since (M) holds, it follows from (FA) that $f$ has a fixed apex $u \in \operatorname{Sub} f(P)$. Then $u=f(v)$ and $v=f(u)$. From inequality (N) we have the following inequalities: $u \leq f(u)=v \leq f(v)=u$, i.e., $u=v=f(u)$. Thus $f$ has fixed point. 
$(\mathrm{FP}) \Rightarrow(\mathrm{ZT})$. Since $P$ is chain complete, Sub $f(P)$ is an inductive poset. Applying (FP) to the set $\operatorname{Sub} f(P)$, we obtain that $f$ has a fixed point.

$(Z T) \Rightarrow(Z L)$ (Proof sketch). Suppose that $(Z L)$ is false. Let $L_{0}$ be the family of all nonempty linearly ordered subsets of $P$ and let $L=L_{0} \cup\{\varnothing\}$. The family $L$ is partially ordered by the inclusion relation $\subset$ between subsets of $P$. For each $A \in L_{0}$ the set

$$
A_{U}=\{x \in P: x \text { is an upper bound for } A \text { and } x \notin A\}
$$

is nonempty because if $x$ is an upper bound for $A$ and $y \in P$ is such that $x \leq y$ and $x \neq y$ then $u \in U_{A}$. Let $U_{\varnothing}=\left\{x_{0}\right\}$, where $x_{0}$ is an arbitrary element of $P$.

Let $f$ be a function defined on $L$ such that $f(A)=A \cup\left\{g\left(U_{A}\right)\right\}$ for all $A \in L$, where $g$ is the identity mapping $g(x)=x$ with domain $\left\{U_{A}: A \in L\right\}$.

Now $L$ satisfies the condition of chain completeness of (ZT). By definition of $f$ we have $A \subset f(A)$ so that condition (b) of (ZT) is satisfied, as well as conditions (a) and (c); but $f$ has no fixed point, i.e., we have a contradiction. This completes the proof.

A brief and simpler proof of the statement that $(\mathrm{ZT})$ implies $(\mathrm{ZL})$ may be found in [RR2, p. 72].

In this connection, we give some new strong forms of (FA) and (FP) from Theorem 1. Also, we present a new characterization of inductiveness (quasiinductiveness, chain-completeness) of posets in terms of fixed apexes.

Our next purpose is to prove a result about partial order relations, now usually called Zorn's lemma, which has far-reaching consequences in several branches of mathematics. We give a strong form of Zorn's lemma due to Bourbaki, for well-ordered $(:=$ wo) chains.

Lemma 1 [Bo]. Let $P$ be a quasi-inductive partially ordered set: Then $P$ has a maximal element.

Let $P$ be a partially ordered set and $f$ and a mapping from $P$ into $P$. For any $f: P \rightarrow P$ it is natural to consider the following set:

$$
\operatorname{Sub}[f(P)):=f(P) \cup\{a \in P \mid a=u b C \text { for some wo chain } C \text { in } f(P)\},
$$

where $u b C$ is an upper bound of well-ordered chain $C$.

Lemma 2 (Fixed Apexes Lemma). Let $P$ be a quasi-inductive (quasi-chain complete) poset and $f$ a mapping from $P$ into $P$ such that

$$
x \leq f^{2}(x) \text { for all } x \in \operatorname{Sub}[f(P)) .
$$

Then $f$ has a fixed apex.

Proof. By Lemma 1 there exists a maximal element $z \in P$; i.e., $\operatorname{Sub}[f(P))$ has a maximal element $z \in \operatorname{Sub}[f(P))$. From condition $\left(\mathbf{M}^{\prime}\right)$ we have $z \leq f^{2}(z)$ and, because $z$ is a maximal element of $\operatorname{Sub}[f(P))$, we have $f^{2}(z) \leq z$. Hence, $f^{2}(z)=z$; so from the preceding remarks, $f$ has a fixed apex.

The lemmas help to obtain the following result. We show later that the existence of a fixed apex for each mapping from a poset into itself that satisfies $\left(\mathbf{M}^{\prime}\right)$ is a necessary and sufficient condition for the poset to be quasi-inductive. 
Theorem 2. If $P$ is a poset such that every $f: P \rightarrow P$ that satisfies $\left(\mathbf{M}^{\prime}\right)$ has a fixed apex, then $P$ is quasi-inductive. (The Axiom of Choice is not used in the proof.)

Proof. We have to show that under the assumption that the poset $P$ is not quasi-inductive there exists a mapping $f$ on $P$ to $P$ with condition $\left(M^{\prime}\right)$ and without fixed apexes.

Suppose $P$ is not quasi-inductive. Then there exists a well-ordered chain $C$ in $P$ that has no upper bound. Let $U$ be a chain cofinal with $C$ such that

$$
U:=\left\{x \in C \mid x_{0} \leq x\right\}, \quad x_{0}:=\text { a fixed element of } C=\min U .
$$

Thus all elements of $U$ can be arranged in a sequence, i.e., one can show that there exists a strictly increasing sequence $\left\{x_{\alpha}\right\}$ in $U$ such that its upper bound does not exist.

We define a mapping $f$ from $P$ into itself by

$$
f(x)= \begin{cases}x_{\alpha+1} & \text { if } x=x_{\alpha} \in U, \\ x_{0}:=\min U, & x \notin U,\end{cases}
$$

where $x_{\alpha} \leq x_{\alpha+1}\left(x_{\alpha} \neq x_{\alpha+1}\right)$ for any $\alpha<w$, and where $w$ is any ordinal. Now, for any $x \in U(\supset \operatorname{Sub}[f(P)))$ we have $x \leq f^{2}(x)$, i.e., $x=x_{\alpha} \leq x_{\alpha+2}=$ $f\left(x_{\alpha+1}\right)=f\left(f\left(x_{\alpha}\right)\right)=f^{2}\left(x_{\alpha}\right)=f^{2}(x)$ for $\alpha<w$, so $f$ satisfies $\left(\mathbf{M}^{\prime}\right)$ and does not have a fixed apex.

The following result of Bourbaki [Bo] allows us to prove the basic fixpoint theorem for complete posets.

Lemma 3. Let $P$ be a chain complete poset and $f: P \rightarrow P$ a map such that $x \leq f(x)$ for all $x \in P$. Then $f$ has a fixpoint.

Our next statement extends this lemma to quasi-inductive posets.

Lemma 4 (Fixed Point Lemma). Let $P$ be a quasi-inductive poset and $f: P \rightarrow P$ a map such that

$$
x \leq f(x) \text { for all } x \in \operatorname{Sub}[f(P)) .
$$

Then there exists a fuxed point of $f$.

A brief proof of this statement based on Bourbaki's lemma may be found in [Ta3].

We say that $P$ has the quasi-general fixed point property if every map $f$ of $P$ into itself with condition $\left(\mathrm{N}^{\prime}\right)$ has a fixed point. Analogously, $P$ is said to have the quasi-fixed apex property if every map $f$ of $P$ into itself with condition $\left(\mathbf{M}^{\prime}\right)$ has a fixed apex.

Theorem 3. Let $P$ be a partially ordered set. Then the following statements are equivalent:

(a) $P$ is quasi-inductive.

(b) $P$ has the quasi-fixed apex property.

(c) $P$ has the quasi-general fixed point property.

Proof. From Theorem 2, (a) is equivalent to (b). Lemma 4 implies that (c) is a consequence of (a). Thus, we need only show that (c) implies (a). 
Suppose $P$ is not quasi-inductive. Then there is a chain $C$ in $P$ that does not have an upper bound. Let $U$ be an ordered chain cofinal with $C$. Define a mapping $f$ from $P$ into itself by (1). Then $f$ is well defined and for any $x \in \operatorname{Sub}[f(P))$ we have $x \leq f(x)$, i.e., $x=x_{\alpha} \leq x_{\alpha+1}=f\left(x_{\alpha}\right)=f(x)$. Thus, $f$ satisfies $\left(\mathrm{N}^{\prime}\right)$ and does not have a fixed point.

Theorem 4. If $P$ is a poset such that every mapping $f: P \rightarrow P$ that satisfies (M) has a fixed apex, then $P$ is quasi-inductive. (The Axiom of Choice is not used in the proof.)

The proof is analogous to the proof of the preceding statements.

As indicated in [Ta2], $P$ is said to have the general fixed point property if every map $f$ of $P$ into itself with the condition (N) has a fixed point. Analogously, $P$ is said to have the fixed apex property if every map $f$ of $P$ into itself with the condition $(\mathbf{M})$ has a fixed apex. The following theorem summarizes things in [Ta2] and in preceding statements.

Theorem 5. Let $P$ be a partially ordered set. Then the following statements are equivalent:

(a) $P$ is inductive.

(b) $P$ is quasi-inductive.

(c) $P$ has the fixed apex property.

(d) $P$ has the general fixed point property.

(e) $P$ has the quasi-fixed apex property.

(f) $P$ has the quasi-general fixed point property.

We note, as an immediate application of the preceding statement (equivalents of inductiveness and quasi-inductiveness), that Zorn's lemma and Bourbaki's lemma are equivalent.

Let $P$ be a partially ordered set and $f$ a mapping from $P$ into $P$. For any $f: P \rightarrow P$ it is natural to consider the set

$$
\overline{f(P)}:=f(P) \cup\{a \in P \mid a=\sup C \text { for some chain } C \text { in } f(P)\} .
$$

Analogously, Bourbaki's Lemma, Fixed Apexes Lemma, and Fixed Point Lemma are equivalent. Also, we have the following statement as a consequence.

Theorem 6 (Well-Ordering Principle). Let $P$ be a set partially ordered by an ordering relation $\leq$. Then the following statements are equivalent:

(a) Bourbaki's Lemma.

(b) Let $P$ be a quasi-chain complete poset. Then $P$ has a maximal element.

(c) Let $P$ be a chain complete and $f$ a mapping form $P$ into itself such that

$$
x \leq f^{2}(x) \quad \text { for all } x \in \overline{f(P)} .
$$

Then $f$ has a fixed apex.

(d) Let $P$ be chain complete and $f$ a mapping from $P$ into itself such that

$$
x \leq f(x) \quad \text { for all } x \in \overline{f(P)} .
$$

Then $f$ has a fixed point. 
Based on Theorem 1 and the above facts, we prove

Theorem 7 (Local forms). Let $P$ be a partially ordered set with ordering relation $\leq$. Then the following statements are equivalent:

(a) Zorn's lemma.

(b) Let $P$ be a quasi-chain complete poset, and $f$ an increasing mapping from $P$ into $P$ such that

$$
a \leq f^{2}(a) \quad \text { for some } a \in P .
$$

Then $f$ has a fixed apex.

(c) [Ta3] Let $P$ be a quasi-chain complete poset, and $f$ an increasing mapping from $P$ into $P$ such that

$$
a \leq f(a) \quad \text { for some } a \in P .
$$

Then $f$ has a fixed point.

Proof. (a) $\Rightarrow(\mathrm{b})$. Consider the subset $A$ of $X$ given by $A:=\{x \in X: x \leq$ $\left.f^{2}(x)\right\}$. From this condition we see that $A$ is nonempty. Since $x \leq f^{2}(x)$ implies $f(x) \leq f^{2}(f(x))$, and we therefore have that $f$ maps $A$ into $A$. Next, let $C$ be a nonempty well-ordered chain of $A$ and $\xi$ a least upper bound of $C$. Also, since $x \leq \xi$ for every $x \in C$, it follows that $f^{2}(x) \leq f^{2}(\xi)$ for every $x \in C$. However, since $C$ is a subset of $A$, it follows that $x \leq f^{2}(x) \leq f^{2}(\xi)$ for every $x \in C$. So $f^{2}(\xi)$ is an upper bound of $C$ and, consequently, $\xi \leq f^{2}(\xi)$. It then follows that $A$ is a nonempty poset with the property that each nonempty well-ordered chain of $A$ has an upper bound, i.e., $A$ is a quasiinductive set and $f$ maps $A$ into $A$; thus, according to (FA) of Theorem 1 , we see that $f$ has a fixed apex, as desired.

The proofs for (b) $\Rightarrow(\mathrm{c})$ and $(\mathrm{c}) \Rightarrow(\mathrm{a})$ are analogous to the proofs of the statements $(\mathrm{FA}) \Rightarrow(\mathrm{FP})$ and $(\mathrm{ZT}) \Rightarrow(\mathrm{ZL})$ of Theorem 1 .

An immediate corollary of the preceding Fixed Point Lemma and its proof is

Theorem 8. Let $F$ be a family of mappings of a poset $P$ into itself such that

$$
x \leq f(x) \text { for all } x \in P \text {, all } f \in F .
$$

If $P$ is a quasi-inductive poset, then the family $F$ has a common fixed point for all $f \in P$.

As proved in the author's Thesis [Ta1], the following statements are equivalent: Zorn's lemma, (ZT), and Theorem 8. (Also see [Ta3].) A special case of the above statement can be found in [RR2]. They credit Abian [A], who formulated a fixed point theorem equivalent to Zorn's lemma.

An immediate corollary of the above Fixed Point Lemma is the following equivalent form of the Axiom of Choice.

Theorem 9. Let $F$ be a family of mappings of a poset $P$ into itself such that

$$
x \leq f^{2}(x) \text { for all } x \in \operatorname{Sub}[f(P)) \text {, all } f \in F .
$$

If $P$ is a quasi-inductive poset, then the family $F$ has a common fixed apex for all $f \in F$.

The proof is analogous to the proof of the preceding statement. 
In concluding, we note that the fixed apex theorems can easily be generalized from $f^{2}$ to $f^{n}:=f\left(f^{n-1}\right)$ for $n \geq 2$.

The conception of apexes can be broadened from $f^{2}$ to $f^{n}$ for some positive integer $n \geq 2$. The points $u_{1}, \ldots, u_{n} \in P$ are called fixed apexes of $f: P \rightarrow P$ if

$$
u_{1}=f\left(u_{2}\right), u_{2}=f\left(u_{3}\right), \ldots, u_{n-1}=f\left(u_{n}\right), u_{n}=f\left(u_{1}\right)
$$

or if

$$
u_{2}=f\left(u_{1}\right), u_{3}=f\left(u_{2}\right), \ldots, u_{n}=f\left(u_{n-1}\right), u_{1}=f\left(u_{n}\right)
$$

for some positive integer $n \geq 2$.

We note that the map $f$ has fixed apexes $u_{1}, \ldots, u_{n} \in P$ if and only if $f^{n}$ for $n \geq 2$ has a fixed point. For if $f$ has fixed apexes $u_{1}, \ldots, u_{n} \in P$ then $u_{1}=f\left(u_{2}\right)=f^{2}\left(u_{3}\right)=\cdots=f^{n-1}\left(u_{n}\right)=f^{n}\left(u_{1}\right)$, or $u_{n}=f\left(u_{n-1}\right)=$ $\cdots=f^{n-1}\left(u_{1}\right)=f^{n}\left(u_{n}\right)$, so $f^{n}$ has a fixed point. On the other hand, if the equation $x=f^{n}(x)$ has a solution $\xi=f^{n}(\xi)$ for some $\xi \in P$, then $f$ has fixed apexes $\xi, f^{n-1}(\xi), f^{n-2}(\xi), \ldots, f(\xi)$ because $\xi=f^{n}(\xi), f^{n-1}(\xi)=$ $f\left(f^{n-2}(\xi)\right), \ldots, f(\xi)=f(\xi)$.

It will be observed that all results for fixed apexes from this paper and from [Ta2] hold for the generalizations of fixed apexes given by $(A L)$ and $(A R)$. We state only the following basic result, which is also an equivalent form of the Axiom of Choice.

Lemma 5. Let $P$ be a quasi-inductive poset, and $f$ a mapping from $P$ into $P$ such that for some positive integer $n \geq 2$,

$$
x \leq f^{n}(x) \quad \text { for all } x \in \operatorname{Sub}[f(P)) .
$$

Then there exist fixed apexes of $f$.

The proof is analogous to the proof of Lemma 2.

\section{REFERENCES}

[A] A. Abian, A fixed point theorem equivalent to the axiom of choice, Abstracts Amer. Math. Soc. 4 (1983), no. 388.

[Bi] G. Birkhoff, Lattice theory, Amer. Math. Soc. Colloq. Publ., vol. 25, Amer. Math. Soc., Providence, RI, 1948.

[Bo] N. Bourbaki, Sur le theoreme de Zorn, Arch. Math. 2 (1950), 434-437.

[G] K. Godel, The consistency of the continum hypothesis, Ann. of Math. Stud., vol. 3, Princeton Univ. Press, Princeton, NJ, 1940, p. 66.

[J] T. J. Jech, The Axiom of Choice, North-Holland, Amsterdam, 1973.

[Ka] C. Kuratowski, Une methode delimination des nombers transfinies raisonnements mathematiques, Fund. Math. 3 (1922), 76-108.

[Ke] G. Kurepa, Sur la relation d'inclusion et Taxiome de choix de Zermelo, Bull. Soc. Math. France 80 (1952), 225-232.

[Mos] A. Mostowski, On the principle of dependent choices, Fund. Math. 35 (1948), 127-130.

[Mo] G. H. Moore, Zermelo's Axiom of Choice, its origins, development and influence, SpringerVerlag, New York, Heidelberg, and Berlin, p. 410.

[R] H. Rubin, On a problem of Kurepa concerning the axiom of choice, Notices Amer. Math. Soc. 5 (1958), no. 378. 
[RR1] H. Rubin and J. Rubin, Equivalents of the Axiom of Choice. North-Holland, Amsterdam and London, 1970, p. 134.

[RR3] - Some new forms of the axiom of choice, Notices Amer. Math. Soc. 7 (1960), no. 380.

[RR2] _ Equivalents of the Axiom of Choice. II, North-Holland, Amsterdam, New York, and Oxford, 1985, p. 322.

[Ta1] M. Tasković, Banach's mappings of fixed points on spaces and ordered sets, Thesis, Math. Balkanica 8 (1978), 150.

[Ta3] On an equivalent of the axiom of choice and its applications, Math. Japonica 31 (1986), 979-991.

[Ta2] Characterizations of inductive posets with applications, Proc. Amer. Math. Soc. 104 (1988), 650-660.

[Z] E. Zermelo, Neuer Beweis fur die Moglichkeit einer Wohlordnung, Math. Ann. 15 (1908), 107-128.

[Zo] Max Zorn, A remark on method in transfinite algebra, Bull. Amer. Math. Soc. 41 (1935), 667-670.

Prirodno-matematički fakultet, University of Beograd, 11000 Beograd, P. O. Box 550, YugosLavia 